\title{
On Simple GP - Injective Modules
}

Mohammed Th. Youns

College of Engineering

University of Mosul

Received on: 07/03/2012
Najlaa T. Jassim

College of Basic Education

University of Mosul

Accepted on: 19/04/2012

\begin{abstract}
In this paper, we study rings whose simple right R-module are GP-injective. We prove that ring whose simple right R-module is GP-injective it will be right $s \pi$-weakly regular ring. Also, proved that if $\mathrm{R}$ is $\mathrm{N}$ duo ring or $\mathrm{R}$ is $\mathrm{NCI}$ ring whose simple right $\mathrm{R}-$ module is GP-injective is S-weakly regular ring.

Keywords: Modules, weak regular rings, N duo ring, NCI ring.

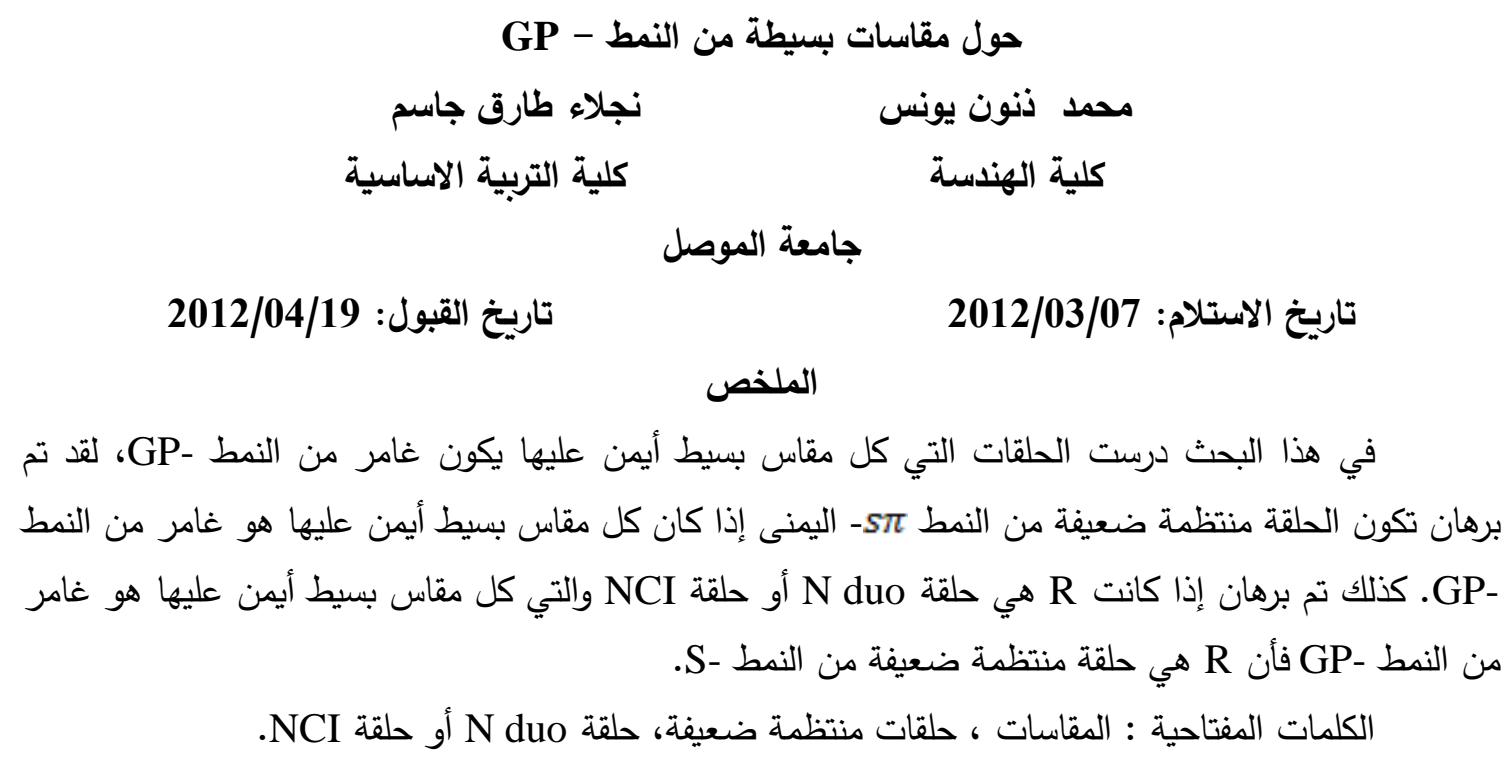

\section{1- Introduction}

Throughout in this paper, $\mathrm{R}$ is associative ring with identity and all modules are unitary. For a subset $X$ of $R$, the left(right) annihilator of $X$ in $R$ is denoted by $1(X)(r(X))$. If $X=\{a\}$, we usually abbreviate it to $1(a)(r(a))$. We write $J(R), N(R)$, $N^{*}(R), P(R)$ for the Jacobson radical, the set of nilpotent elements, the nil radical (that means the sum of all nil ideals), prime radical (that means the intersection of all prime ideals)respectively. $\quad N_{2}(R)=\left\{a \in R / a^{2}=0\right\}$. A ring $\mathrm{R}$ is called NI if $N^{*}(R)=N(R)$ [9]. A ring $\mathrm{R}$ is 2-primal if $N(R)=P(R)$ [2]. A ring $\mathrm{R}$ is said to be semiprimitive if $\mathrm{J}(\mathrm{R})=0$ [1]. An element $a$ in the ring $\mathrm{R}$ said to be right (left) weakly regular if $a \in \operatorname{aRaR}(a \in R a R a)$ [12].

A right $\mathrm{R}$-module $\mathrm{M}$ is called Generalized Principally injective (briefly, GPinjective) if for any $a \in R$, there exists a positive integer $\mathrm{n}$ such that $a^{n} \neq 0$ and any right $\mathrm{R}$-homomorphism of $a^{n} R$ into $\mathrm{M}$ extends to one of $\mathrm{R}$ into $\mathrm{M}$ [8]. Right GPinjective modules are called right YJ-injective modules by several authors [16]. 


\section{Some Properties of Rings whose Simple Right R-module are GP-injective.}

We give a different prove that proved by $\mathrm{Kim}$, et. al. in [8].

\section{Theorem 2.1}

Let $R$ be a ring whose every simple right $R$-module is GP-injective. Then $R$ is semiprime.

\section{Proof:}

We shall show that is no nilpotent ideal in $R$, if not, suppose there exists $0 \neq a \in R$ with $(a R)^{2}=0, a R a R=0$, that means $R a R \subseteq r(a)$, there exists a maximal right ideal $\mathrm{M}$ of $\mathrm{R}$ containing $\mathrm{r}(\mathrm{a}), \mathrm{R} / \mathrm{M}$ is GP-injective. Hence, there exists an appositive integer $\mathrm{n}=1$ such that $a \neq 0$ and any $\mathrm{R}$-homomorphism of $a \mathrm{R}$ into $\mathrm{R} / \mathrm{M}$ extends to one of $\mathrm{R}$ into $\mathrm{R} / \mathrm{M}$, we define $f: a R \rightarrow R / M$ such that $f(a r)=r+M$ where $r \in R$. We have to show that $\mathrm{f}$ is well defined R-homomorphism, let $\mathrm{ax}=$ ay where $x, y \in R, \mathrm{a}(\mathrm{x}-\mathrm{y})=0,(x-y) \in r(a) \subseteq M, \quad \mathrm{x}+\mathrm{M}=\mathrm{y}+\mathrm{M}, \mathrm{f}(\mathrm{ax})=\mathrm{x}+\mathrm{M}=\mathrm{y}+\mathrm{M}=\mathrm{f}(\mathrm{ay})$, $\mathrm{f}(\mathrm{ax})=\mathrm{f}(\mathrm{ay})$, so $\mathrm{f}$ is well defined right R-homomorphism, since R/M is GP-injective, there exists $b+M \in R / M \quad$ such that $1+\mathrm{M}=\mathrm{f}(\mathrm{a})=(\mathrm{b}+\mathrm{M})(\mathrm{a}+\mathrm{M})=\mathrm{ba}+\mathrm{M}, \quad 1+\mathrm{M}=\mathrm{ba}+\mathrm{M}$,

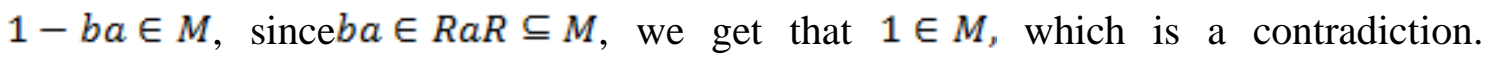
Therefore, $\mathrm{a}=0$. This shows that $\mathrm{R}$ issemiprime.

We give a different prove that is proved by Xue in [16].

\section{Proposition 2.2}

Let $R$ be a ring whose simple right $R$-module is GP-injective. Then, $R$ is semiprimitive.

\section{Proof:}

We shall show that $J(R)=0$,if not, there exists $0 \neq a \in J(R)$, then either $R a^{n} R+r\left(a^{n}\right)=R$, or not, if not, there exists a maximal right ideal $\mathrm{M}$ of $\mathrm{R}$ containing $R a^{n} R+r\left(a^{n}\right)_{3} \mathrm{R} / \mathrm{M}$ is GP-injective, there exists a positive integer $\mathrm{n}$ and $a^{n} \neq 0$ such that any R-homomorphism of $a^{n} \mathrm{R}$ into $\mathrm{R} / \mathrm{M}$ extends to one of $\mathrm{R}$ into $\mathrm{R} / \mathrm{M}$, Let $f: a^{n} R \rightarrow R / M$ such that $f\left(a^{n} r\right)=r+M$, where $r \in R$, we have to show that $\mathrm{f}$ is well defined, let $a^{n} x=a^{n} y$ where $x, y \in R, a^{n}(x-y)=0,(x-y) \in r\left(a^{n}\right) \in M_{\text {, }}$ then $(\mathrm{x}-\mathrm{y})+\mathrm{M}=\mathrm{M}, \mathrm{x}+\mathrm{M}=\mathrm{y}+\mathrm{M}, \mathrm{f}\left(a^{n} \mathrm{x}\right)=\mathrm{x}+\mathrm{M}=\mathrm{y}+\mathrm{M}=\mathrm{f}\left(a^{n} \mathrm{y}\right), \mathrm{f}\left(a^{n} \mathrm{x}\right)=\mathrm{f}\left(a^{n} \mathrm{y}\right)$, so $\mathrm{f}$ is well defined right $\mathrm{R}$-homomorphism, since $\mathrm{R} / \mathrm{M}$ is GP-injective, there exists $b+M \in R / M$ such that $1+\mathrm{M}=\mathrm{f}\left(a^{n}\right)=(\mathrm{b}+\mathrm{M})\left(a^{n}+\mathrm{M}\right)=\mathrm{b} a^{n}+\mathrm{M}, \quad 1+\mathrm{M}=\mathrm{b} a^{n}+\mathrm{M}, 1-b a^{n} \in M$, since $b a^{n} \in R a^{n} R \subseteq M$, we get that $1 \in M_{\text {s }}$ which is a contradiction. That means $R a^{n} R+r\left(a^{n}\right)=R$ in particular there exists $y_{y} z \in R$ and $v \in r\left(a^{n}\right)$ such that $y a^{n} z+v=1, a^{n} y a^{n} z+a^{n} v=a^{n}, a^{n} y a^{n} z=a^{n}, a^{n}\left(1-y a^{n} z\right)=0$, since $a \in J(R)$,so $y a^{n} z \in J(R), 1-y a^{n} z$ is invertible, there exists $u \in R_{y}$ such that $\left(1-y a^{n} z\right) u=1, a^{n}=a^{n}\left(1-y a^{n} z\right) u=0 u=0$,must $a^{n}=0 \quad$ which is a contradiction with $a^{n} \neq 0$. Therefore, $a=0$, so, $\mathrm{J}(\mathrm{R})=0$. This shows that $\mathrm{R}$ is semiprimitive.

\section{Corollary $\mathbf{2 . 3}$}

Let $R$ be a ring whose simple right $R$-module is $G P$-injective. Then, $N^{*}(R)=0$.

\section{Proof:}


We shall show that $N^{*}(R)=0$, $\operatorname{since} N^{*}(R)$ is the large nil ideal of R, It is clearly that $\mathrm{J}(\mathrm{R})$ containing every nil ideal, so $N^{*}(R) \subseteq J(R)$, but $J(R)=0$ by Proposition 2.3. Thisshows that $N^{*}(R)=0$.

\section{Theorem 2.4}

Let $R$ be a ring whose simple right $R$-module is GP-injective. Then, the set $\mathrm{N}_{2}(R)$ is right weakly regular.

\section{Proof:}

We shall show that $R b R+r(b)=R$, for all $b \in N_{2}(R)$, if not,suppose there exists $0 \neq a \in N_{2}(R)$, such that $\operatorname{RaR}+r(a) \neq R$, then there exists a maximal right ideal $\mathrm{M}$ of $\mathrm{R}$ containing $\operatorname{RaR}+r(a), \mathrm{R} / \mathrm{M}$ is GP-injective, there exists appositive integer $\mathrm{n}=1$ such that $a \neq 0$ and any R-homomorphism of $a \mathrm{R}$ into R/M extends to one of $\mathrm{R}$ into R/M. Now, let $f: a R \rightarrow R / M$ such that $f(a r)=r+M$ where $r \in R$. Note that $\mathrm{f}$ is well defined right $\mathrm{R}$-homomorphism, since $\mathrm{R} / \mathrm{M}$ is GP-injective there exists $b+M \in R / M$ such that $1+\mathrm{M}=\mathrm{f}(\mathrm{a})=(\mathrm{b}+\mathrm{M})(\mathrm{a}+\mathrm{M})=\mathrm{ba}+\mathrm{M}, 1+\mathrm{M}=\mathrm{ba}+\mathrm{M}, 1-b a \in M$, since $b a \in R a R \subseteq M$, we get that $1 \in M$, which is a contradiction.. Therefore, $R a R+r(a)=R$. In particular, there exists $y, z \in R$ and $v \in r(a)$ such that $y a z+v=1, a y a z+a v=a, a y a z=a$, that is for all $a \in N_{2}(R)$. This shows that the set $N_{2}(R)$ is right weakly regular.

\section{Theorem 2.5}

Let $R$ be a ring without zero divisors whose simple right $R$-module is GPinjective. Then, $R$ is a simple ring.

\section{Proof:}

We shall show that there is no two sided ideal of $R$, if not there exists a two sides ideal of $\mathrm{R}, \mathrm{RaR}$ is a two sided ideal for some $0 \neq a \in R_{\text {s }}$ since $0 \neq a, \mathrm{RaR} \neq 0$, if $R a R \neq R$, there exists a maximal right ideal $\mathrm{M}$ of $\mathrm{R}$ containing $R a R, \mathrm{R} / \mathrm{M}$ is GPinjective, there exists a positive integer $\mathrm{n}$ and $a^{n} \neq 0$ such that any R-homomorphism of $a^{n} \mathrm{R}$ into $\mathrm{R} / \mathrm{M}$ extends to one of $\mathrm{R}$ into $\mathrm{R} / \mathrm{M}$, we define $f: a^{n} R \rightarrow R / M$ such that $f\left(a^{n} r\right)=r+M_{y} \quad$ where $\quad r \in R$, let $\mathrm{r}_{1}, \mathrm{r}_{2} \in R \quad$ such that $a^{n} r_{1}=a^{n} r_{2}, a^{n}\left(r_{1}-r_{2}\right)=0, r_{1}-r_{2} \in r\left(a^{n}\right)=0$, since a is a non-zero divisor, so must $r_{1}=r_{2}, \quad f\left(a^{n} r_{1}\right)=r_{1}+M=r_{2}+M=f\left(a^{n} r_{2}\right)$, so $\quad \mathrm{f} \quad$ is well defined Rhomomorphism, since $\mathrm{R} / \mathrm{M}$ is GP-injective, there exists $b+M \in R / M$ such that $1+\mathrm{M}=\mathrm{f}\left(a^{n}\right)=(\mathrm{b}+\mathrm{M})\left(a^{n}+\mathrm{M}\right) \quad=\mathrm{b} a^{n}+\mathrm{M}, \quad 1+\mathrm{M}=\mathrm{b} a^{n}+\mathrm{M}, \quad$ but $\mathrm{b} a^{n} \in R a R \subseteq \mathrm{M}, 1-b a^{n} \in M, 1 \in M$, which is a contradiction. Therefore, $R a R=R$, for all $a \in R_{y}$ that means $\mathrm{R}$ not containing any two sided ideal of $\mathrm{R}$. This shows that $\mathrm{R}$ a simple ring.

\section{Rings Whose Simple Right R-module are GP-injective and it relation with other Rings.}

In this section, we give different conditions to the ring whose simple right $\mathrm{R}$ module is GP-injective to get the reduced, S-weakly regular, regular, strongly regular ring.

A ring $\mathrm{R}$ is said to be $\mathrm{N}$ duo if $\mathrm{aR}=\mathrm{Ra}$, for all $a \in N(R)$ [15]. 


\section{Theorem 3.1}

Let $R$ be $N$ duo ring whose every simple right $R$-module is GP-injective. Then, $R$ is a reduced ring.

\section{Proof:}

We shall show that $\mathrm{N}(\mathrm{R})=0$, if not, there exists $0 \neq a \in N(R)$ with $a^{2}=0$, if $\mathrm{aR}+\mathrm{r}(\mathrm{a}) \neq R$, there exists a maximal right ideal $\mathrm{M}$ of $\mathrm{R}$ containing $a R+r(a), \mathrm{R} / \mathrm{Mis}$ GP-injective, there exists an appositive integer $\mathrm{n}=1$ such that $a \neq 0$ and any $\mathrm{R}$ homomorphism of $a \mathrm{R}$ into $\mathrm{R} / \mathrm{M}$ that extends to one of $\mathrm{R}$ into $\mathrm{R} / \mathrm{M}$. Let $f: a R \rightarrow R / M$ such that $f(a r)=r+M$ where, $r \in R$. Note that $\mathrm{f}$ is well defined right $\mathrm{R}$ homomorphism, since $\mathrm{R} / \mathrm{M}$ is GP-injective there exists $b+M \in R / M$ such that $1+\mathrm{M}=\mathrm{f}(\mathrm{a})=(\mathrm{b}+\mathrm{M})(\mathrm{a}+\mathrm{M})=\mathrm{ba}+\mathrm{M}, 1+\mathrm{M}=\mathrm{b}+\mathrm{M}, 1-b a \in M$, since $\mathrm{R}$ is $\mathrm{N}$ duo ring and $a \in N(R)$, we get $\mathrm{aR}=\mathrm{Ra}, b a \in R a=a R \subseteq M$, so1 $\in M$, which is a contradiction. Therefore, $a R+r(a)=R$. In particular, there exists $z \in R$ and $v \in r(a)$ such that $a z+v=1, a^{2} z+a v=0=a$, for all $a \in N(R)$, This shows that $R$ is a reduced ring.

Call a ring $\mathrm{R}$ NCI if $\mathrm{N}(\mathrm{R})$ is containing a non-zero ideal of $\mathrm{R}$ whenever $N(R) \neq 0$. Clearly, NI ring is NCI [5].

\section{Theorem 3.2}

Let $R$ be an NCI ring whose simple right $R$-module is GP-injective. then $R$ is a reduced ring.

\section{Proof:}

We shall show that $\mathrm{N}(\mathrm{R})=0$, if not, $0 \neq N(R)$, since $\mathrm{R}$ is an NCI ring, so $\mathrm{N}(\mathrm{R})$ is containing a non-zero ideal $I$, but $I$ is nil ideal, It is clearly that $J(R)$ containing every nil ideal, so $I \subseteq J(R)=0$, form proposition 2.4, I=0, that is mean $N(R)$ mustbe an ideal, similarly $N(R) \subseteq J(R)=0, \mathrm{~N}(\mathrm{R})=0$. This is shows that $R$ is reduced ring.

\section{Theorem 3.3}

Let $R$ be aring whose simple right $R$-module is GP-injective. Then, the following conditions are equivalent:

$1-R$ is reduced ring.

2- $R$ is $N$ duo ring.

3- $R$ is 2-priaml ring.

4- $R$ is NI ring.

5- $R$ is NCI ring.

\section{Proof:}

$1 \rightarrow 2$, is clear and $2 \rightarrow 1$, by Theorem 3.2

$1 \rightarrow 3 \rightarrow 4 \rightarrow 5$, is clearand $5 \rightarrow 1$, by Theorem 3.1

Call a ring $\mathrm{R} S$-weakly regular ring if $a \in a R a^{2} R_{y}$ for all $a \in R$ [14].

\section{Theorem 3.4}

Let $R$ be aring whose simple right $R$-module is GP-injective. Then, $R$ is $S$-weakly regular ring. If satisfies one of the following conditions.

$1-R$ is a reduced ring.

2- $R$ is $N$ duo ring.

3- $R$ is 2-priaml ring. 
4- $R$ is NI ring.

5- $R$ is NCI ring.

\section{Proof:}

We shall prove that $\mathrm{R}$ is $\mathrm{S}$-weakly regular when $\mathrm{R}$ is reduced, and the proof of the other condition that is clearly form Theorem 3.3.

We shall show that $R d^{2} R+r(d)=R$, for all $d \in R$, if not, there exists $0 \neq a \in R_{\text {s }}$ uch that $R a^{2} R+r(a) \neq R$, there exists a maximal right ideal $\mathrm{M}$ of $\mathrm{R}$ containing $R a^{2} R+r(a), \mathrm{R} / \mathrm{M}$ is GP-injective, there exists an appositive integer $\mathrm{n}$ such that $a^{n} \neq 0$ and any R-homomorphism of $a^{n} \mathrm{R}$ into R/M extends to one of $\mathrm{R}$ into R/M. Let $f: a^{n} R \rightarrow R / M$ such that $f\left(a^{n} r\right)=r+M$ where, $r \in R$. let $a^{n} x=a^{n} y$ where $x, y \in R, a^{n}(x-y)=0,(x-y) \in r\left(a^{n}\right)=r(a) \subseteq M$, since $\mathrm{R}$ is a reduced ring, then $(\mathrm{x}-\mathrm{y})+\mathrm{M}=\mathrm{M}, \mathrm{x}+\mathrm{M}=\mathrm{y}+\mathrm{M}, \mathrm{f}\left(a^{n} \mathrm{x}\right)=\mathrm{x}+\mathrm{M}=\mathrm{y}+\mathrm{M}=\mathrm{f}\left(a^{n} \mathrm{y}\right), \mathrm{f}\left(a^{n} \mathrm{x}\right)=\mathrm{f}\left(a^{n} \mathrm{y}\right)$, so $\mathrm{f}$ is well defined right R-homomorphism, since $\mathrm{R} / \mathrm{M}$ is GP-injective there exists $b+M \in R / M$ such that $1+\mathrm{M}=\mathrm{f}\left(a^{n}\right)=(\mathrm{b}+\mathrm{M})\left(a^{n}+\mathrm{M}\right)=\mathrm{b} a^{n}+\mathrm{M}, 1+\mathrm{M}=\mathrm{b}+\mathrm{M}$. Now $\mathrm{b} a^{n} \in R a^{2} R \subseteq \mathrm{M}$, it is true when $n \geq 2, \quad 1-b a^{n} \in M, 1 \in M$, which is a contradiction. Therefore, $R a^{2} R+r\left(a^{n}\right)=R$, for $n \geq 2$. Now, when $\mathrm{n}=1, f: a R \rightarrow R / M, f(a r)=r+M_{y}$ $1+\mathrm{M}=\mathrm{f}(a)=(\mathrm{b}+\mathrm{M})(a+\mathrm{M})=\mathrm{b} a+\mathrm{M}, 1+\mathrm{M}=\mathrm{b} a+\mathrm{M}$, by multiply $\mathrm{a}+\mathrm{M}$ in the left side and

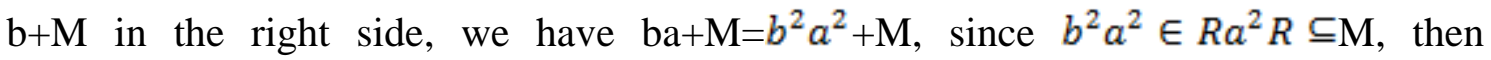
$b a-b^{2} a^{2} \in M$, we get that $b a \in M$, since $1+\mathrm{M}=\mathrm{b} a+\mathrm{M}, 1-\mathrm{ba} \in M, 1 \in M_{y}$ which is a contradiction. Therefore, $R a^{2} R+r(a)=R$, for all $a \in R$. In particular, there exists $\mathrm{y}, z \in R$ and $v \in r(a)$ such that $\mathrm{y}^{2} z+v=1, a y a^{2} z+a v=a y a^{2} z=a$. This shows that $\mathrm{R}$ is an $\mathrm{S}$-weakly regular ring.

An element a of ring $\mathrm{R}$ is said to be a right regular element if the right annihilator ideal is zero $(r(a)=0)$ [6]. A ring $R$ is said to be MERT if and only if every maximal essential right ideal of $R$ is an ideal [14]. A ring $R$ is called Kasch if every simple right $\mathrm{R}$-module embeds in $\mathrm{R}$, equivalently, for every maximal right ideal $\mathrm{M}$ of $\mathrm{R}$ is a right annihilator of $\mathrm{R}$ [3]. Call a ring $\mathrm{R}$ a right SF-ring if each simple right $\mathrm{R}$ module is flat [13]. A ring $\mathrm{R}$ is said to be regular if $a \in a R a$, for all $a \in R$ [11].

\section{Lemma 3.5 [6]}

Let $R$ be a semiprime ring with maximum condition on left and right annihilators. Then, every essential right ideal contains a regular element.

\section{Lemma 3.6 [13]} that $x=y x$.

$R / I$ is right flat $R$-module if and only if for each $x$ in $R$ there is some $y$ in $R$ such

\section{Lemma 3.7 [7]}

Let $R$ be a MERT ring, then the following conditions are equivalent:

$1-R$ is regular.

2- $R$ is right $S F$.

\section{Theorem 3.8}

Let $R$ be a MERT ring whose every simple right module is GP-injective and satisfies maximum condition on left and right annihilators. Then $R$ is Kasch ring and right $S F$-ring, hence $R$ is regular ring.

\section{Proof :}


We shall prove that every maximal right ideal is direct summand, if not, suppose that $\mathrm{M}$ a maximal right ideal of $\mathrm{R}$ which is not a direct summand of $\mathrm{R}$, then $\mathrm{M}$ is a maximal essential right ideal of $\mathrm{R}$, by Theorem 2.1 and Lemma 3.5, we have $\mathrm{M}$ containing a non-zero divisor a, R/M is GP-injective, there exists a positive integer $n$ and $a^{n} \neq 0$ such that any R-homomorphism of $a^{n} \mathrm{R}$ into $\mathrm{R} / \mathrm{M}$ extends to one of $\mathrm{R}$ into $\mathrm{R} / \mathrm{M}$, Let $f: a^{n} R \rightarrow R / M$ such that $f\left(a^{n} r\right)=r+M$, where $r \in R$, since a is a non-zero divisor $\mathrm{f}$ is well defined right $\mathrm{R}$-homomorphism, since R/M is GP-injective, there exists $b+M \in R / M$ such that $1+\mathrm{M}=\mathrm{f}\left(a^{n}\right)=\mathrm{b} a^{n}+\mathrm{M}, 1-b a^{n} \in M$, since $a \in M, \mathrm{M}$ is essential right ideal and $\mathrm{R}$ is MERT ring, $\mathrm{M}$ is an ideal, so $b a^{n} \in M$, we get that $1 \in M_{\text {, }}$ which is a contradiction. Therefore, $M$ a direct summand. This shows that every maximal right ideal of $\mathrm{R}$ is a direct summand.

There exists $\mathrm{J}$ right ideal for any maximal right ideal $\mathrm{M}$ such that $M \oplus J=R$, in particular there exists $m \in M$ and $j \in J$ such that $\mathrm{m}+\mathrm{j}=1$, so for all $d \in M, \mathrm{jd}=0$, then $M \subseteq r(j)$, but $\mathrm{M}$ is a maximal right ideal, we have $\mathrm{M}=\mathrm{r}(\mathrm{j})$, for every maximal right ideal, This shows that $\mathrm{R}$ is a right kasch ring.

Also, md=d for all $d \in M$, from Lemma 3.6, we get that $\mathrm{R} / \mathrm{M}$ is flat right $\mathrm{R}$ module and that is for all $\mathrm{M}$ maximal right ideal of $\mathrm{R}$. This shows that $\mathrm{R}$ is a right SFring.

Since, $\mathrm{R}$ is MERT and right SF-ring, by using Lemma 3.7, we get that $\mathrm{R}$ is a regular ring.

\section{Theorem 3.9}

Let $R$ be $N$ duo ring and MERT whose simple right $R$-module is GP-injective. Then, $R$ is a strongly regular ring.

\section{Proof:}

We shall show that $d R+r(d)=R$, for all $d \in R$. If not then there exists $a R+r(a) \neq R$, for some $a \in R$, there exists a maximal right ideal $\mathrm{M}$ of $\mathrm{R}$ containing $a R+r(a) \cdot \mathrm{M}$ is either essential or direct summand, if $\mathrm{M}$ is not essential, then $\mathrm{M}=\mathrm{r}(\mathrm{e})$ for some $0 \neq e=e^{2} \in R$, by Theorem 3.1, $\mathrm{R}$ is a reduced ring, $a \in r(e)=l(e), a e=0, e \in r(a) \subseteq r(e), \mathrm{e} \in r(e), e^{2}=0$, but $e=e^{2}{ }_{\text {, hence }} \mathrm{e}=0$, and $\mathrm{M}==\mathrm{r}(\mathrm{e})=\mathrm{r}(0)=\mathrm{R}, \mathrm{M}=\mathrm{R}$, which is a contradiction. Therefore, $\mathrm{M}$ is an essential right ideal of $\mathrm{R}$. Thus, $\mathrm{R} / \mathrm{M}$ is GP-injective, there exists a positive integer $\mathrm{n}$ such that $a^{n} \neq 0$ and any R-homomorphism of $a^{n} \mathrm{R}$ into $\mathrm{R} / \mathrm{M}$ extends to one of $\mathrm{R}$ into $\mathrm{R} / \mathrm{M}$, Let $f: a^{n} R \rightarrow R / M$ be defined by $f\left(a^{n} r\right)=r+M$, where $r \in R$. Note that $\mathrm{f}$ is well defined right $\mathrm{R}$-homomorphism, because $\mathrm{R}$ is a reduced ring. since $\mathrm{R} / \mathrm{M}$ is GP-injective, there exists $b+M \in R / M$ such that $1+\mathrm{M}=\mathrm{f}\left(a^{n}\right)=\mathrm{b} a^{n}+\mathrm{M}, 1-b a^{n} \in M$, since $a \in M_{\text {, }}$ $\mathrm{M}$ is an essential right ideal and $\mathrm{R}$ is MERT ring, $\mathrm{M}$ is an ideal, so $b a^{n} \in M$, we get that $1 \in M$, which is also contradiction. Therefore, $a R+r(a)=R$, for all $a \in R$. This shows that $\mathrm{R}$ is a strongly regular ring.

Finally, we give the following important result.

In [16] Xue proved, if every simple left R-module is GP-injective, then for any nonzero $a \in R_{y}$ there exists a positive integer $\mathrm{n}=\mathrm{n}(\mathrm{a})$ such that $a^{n} \neq 0$ and $R a R+l\left(a^{n}\right)=R[$ Proposition 2]. 
In the above proof, we have $R a R+l\left(a^{n}\right)=R$, and $R a^{n} R+l\left(a^{n}\right)=R$, it is clear that proof $R a^{n} R+l\left(a^{n}\right)=R$ leads us to $R a R+l\left(a^{n}\right)=R$ because $R a^{n} R \subseteq R a R$, we give a new proof that strengthens the above $R a^{2 n} R+r\left(a^{n}\right)=R$, hence $R a^{2 n} R \subseteq R a^{n} R \subseteq R a R$.

A ring $\mathrm{R}$ is said to be right (left) st-weakly regular ring if, for every $a \in R$, there exists a positive integer $n$, depending on $a$ such that $a^{n} \in a^{n} R a^{2 n} R\left(a^{n} \in R a^{2 n} R a^{n}\right)[10]$.

\section{Theorem 3.10}

Let $R$ be a ring whose simple right $R$-module is GP-injective. Then $R$ is right s $\pi$-weakly regular ring.

\section{Proof:}

Let $a \in R_{\text {y }}$ and $a$ is not a nilpotent element, if $R a^{2 n} R+r\left(a^{n}\right) \neq R$, then there exists a maximal right ideal $M$ of $R$ containing $R a^{2 n} R+r\left(a^{n}\right)$, by hypothesis $R / M$ is $G P$ - injective, we define $f: a^{n} R \rightarrow R / M$ such that $f\left(a^{n} r\right)=r+M_{y}$ where $r \in R$, we show that $\mathrm{f}$ is well defined, let $a^{n} x=a^{n} y$ where $x, y \in R, a^{n}(x-y)=0$, $(x-y) \in r\left(a^{n}\right) \in M$, then $\quad(\mathrm{x}-\mathrm{y})+\mathrm{M}=\mathrm{M}, \quad \mathrm{x}+\mathrm{M}=\mathrm{y}+\mathrm{M}, \quad \mathrm{f}\left(a^{n} \mathrm{x}\right)=\mathrm{x}+\mathrm{M}=\mathrm{y}+\mathrm{M}=\mathrm{f}\left(a^{n} \mathrm{y}\right)$, $\mathrm{f}\left(a^{n} \mathrm{x}\right)=\mathrm{f}\left(a^{n} \mathrm{y}\right)$. So $\mathrm{f}$ is well defined right R-homomorphism, since R/M is GP-injective, there exists $b+M \in R / M$ and $a^{n} \neq 0$ such that $1+\mathrm{M}=\mathrm{f}\left(a^{n}\right)=(\mathrm{b}+\mathrm{M})\left(a^{n}+\mathrm{M}\right)=\mathrm{b} a^{n}+\mathrm{M}$, $1+\mathrm{M}=b a^{n}+\mathrm{M}, 1-b a^{n} \in M$, since $1+\mathrm{M}=\mathrm{b} a^{n}+\mathrm{M}$, multiply by $a^{n}+M$ from left and $\mathrm{b}+\mathrm{M}$ from right, we get $b a^{n}+\mathrm{M}=b^{2} a^{2 n}+\mathrm{M}, \quad b a^{n}-b^{2} a^{2 n} \in M$, but $b^{2} a^{2 n} \in R a^{2 n} R \subseteq M$, so $b a^{n} \in M$, but $1-b a^{n} \in M$, we get that $1 \in M$, which is a contradiction. Therefore, $R a^{2 n} R+r\left(a^{n}\right)=R$. In particular, there exists $y, z \in R$ and $v \in r(a)$ such that $y a^{2 n} z+v=1, a^{n} y a^{2 n} z+a^{n} v=a^{n}, a^{n} y a^{2 n} z=a^{n}$, and that is for all $a \in R$ which is not nilpotent elements. When $a$ is nilpotent element, there exists a positive integer $m$ such that $a^{m}=0$, so $a^{m}=a^{m} r a^{2 m} s$, for any $r_{s} s \in R$. This shows that $R$ is a right s $\pi$-weakly regular ring. 


\section{REFERENCES}

[1] Anderson, F. W. and Fuller, K. R. (1992),"Ring and Categories of Modules", Graduate Texts in Math. No. 13, Springer-Verlag, New Yourk.

[2] Birkenmeier, G. F. Heatherly, H. E. and Lee, E. K. (1993), Completely prime ideals and associated radicals, Proc. Biennial Ohio State-Denison Conference 1992, edited by S. K. Jain and S. T. Rizvi, World Scientific, Singapore, New Jersey -London-Hong Kong, pp. 102-129.

[3] Faith, C. (1982), Injective modules and injective quotient rings, Lecture Notes in Pure and Appl. Math.,Vol. 72.

[4] Gupta,V. (1984), A generalization of strongly regular rings,Acta. Math. Hung., Vol. 43, No. (1-2), pp. 57-61.

[5] Hwang, S.U. , Jeon, Y. C., and Park, K. S. (2007), On NCI rings, Bull. Korean Math. Soc., Vol. 44, No. 2, pp. 215-223.

[6] Johnson, R. E. and Levy, L. S. (1968), Regular element in semiprime rings, Proceedings of the American Mathematical Society, Vol. 19, No.4, pp. 961-963.

[7] Jule, Z. and Xianneng, D. (1993), Von Neumann regularity of SF-rings, Communications in Algebra, Vol. 21, No. 7, pp. 2445-2451.

[8] Kim, N. K. Nam, S. B. and Kim, J. Y. (1995) " On simple GP-injective modules" Comm. Algebra, Vol. 23, No. 14, pp. 5437-5444.

[9] Lee, Y. and Huh, C. (1998), A note on $\pi$-regular rings, KyunGpook Math. J., Vol. 38, pp. 157-161.

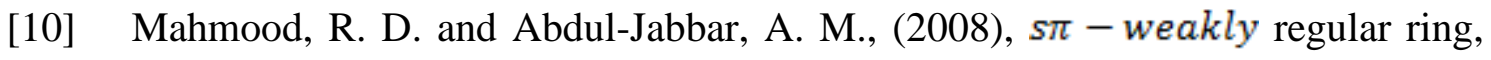
Raf. J. of Comp. Sc. and Math's., Vol. 5, No. 2, p.p.39-46.

[11] Neumann, J. V. (1936), On regular rings, Princeton N. J., Vol. 22, pp. 707-713.

[12] Ramamurhi, V. S. (1973), Weakly regular ring,Canda. Math. Bull., Vol. 16, No. 3, pp.317-321.

[13] Ramamurthi, V. S. (1975), On the injectivity and flatness of certain cyclic modules, Proc. Amer. Math. Soc., Vol. 48, No.1, pp. 21-25.

[14] Yue Chi Ming, R. (1980), On V-rings and prime rings, J. of Algebra, Vol. 62, pp. 13-20.

[15] Wei, J. and Li, L. (2010), Nilpotent elements and reduced rings*, Turk. J. Math., Vol. 34, pp. 1-13.

[16] Xue, W. (1998),A note on YJ-injectivity, Riv. Mat. Univ. Parma, Vol. 6, No. 1, pp. 31-37. 